\title{
Family Environment Is Associated with Endemic Burkitt Lymphoma: A Population-based Case-control Study
}

\author{
Jeanette J. Rainey, Rosemary Rochford, Peter O. Sumba, Dickens Kowuor, Mark L. Wilson, and Ann M. Moormann* \\ Department of Epidemiology, School of Public Health, University of Michigan, Ann Arbor, Michigan; \\ Department of Microbiology and Immunology, SUNY Upstate Medical University, Syracuse, New York; \\ Center for Vector Biology and Control Research, Kenya Medical Research Institute, Kisumu, Kenya; \\ Center for Global Health and Diseases, School of Medicine, Case Western Reserve University, Cleveland, Ohio
}

\begin{abstract}
Endemic Burkitt's lymphoma (eBL) has been linked to Epstein-Barr virus and holoendemic Plasmodium falciparum malaria. These co-infections, however, are insufficient to explain the non-random occurrence of Endemic Burkitt's lymphoma within Equatorial Africa. To explore whether this distribution could be explained by household characteristics and family environment, we conducted a case-control study using 41 hospitalized incident endemic Burkitt's lymphoma cases and 91 healthy controls identified through a population-based multistage cluster-sampling scheme in Nyanza Province, Kenya. In a multivariate analysis, odds ratios associated with having one, two, and three or more younger siblings compared with none were 0.28 (90\% CI: 0.09, 0.83), 0.59 (90\% CI: 0.16, 2.23) and 0.15 (90\% CI: $0.03,0.67)$ respectively, suggesting that children with endemic Burkitt's lymphoma were more likely than controls to be last-born. Children with endemic Burkitt's lymphoma were also more likely to live in non-monogamous families $(\mathrm{OR}=3.12,90 \% \mathrm{CI}: 1.19,8.17)$ and to have at least one deceased parent $(\mathrm{OR}=3.38,90 \% \mathrm{CI}: 1.18,9.64)$. Household characteristics, especially sibship relationships, may contribute to endemic Burkitt's lymphoma and therefore warrant further study.
\end{abstract}

\section{INTRODUCTION}

Endemic Burkitt's lymphoma (eBL) ranks as the most common childhood cancer in sub-Saharan Africa. In high-risk regions, the annual incidence rate of this cancer ranges from one to over seven cases per 100,000 population. Increased risk for eBL is associated with both early infection with the Epstein Barr Virus (EBV) and exposure to holoendemic malaria transmission. ${ }^{1-3}$ However, the ubiquitous distribution of early EBV infection ${ }^{4,5}$ and the non-random occurrence of eBL within holoendemic malaria regions ${ }^{6-9}$ suggest that other cofactors contribute to the etiology of this cancer. ${ }^{6,8,10-14}$ These additional co-factors may involve other environmental or socio-cultural risks, including household and sibship characteristics.

Sibship size and birth order have been linked to an increased risk for certain childhood leukemias and lymphomas in developed countries. ${ }^{15}$ Whether birth order and other familial characteristics are also associated with eBL is currently unknown, in part due to methodological limitations and inadequate cancer surveillance systems. Population-based cancer case-control studies in sub-Saharan Africa are rare, and only one study has been conducted to examine other eBL cofactors. ${ }^{14}$ Cancer registries are incomplete within many countries in Africa, limiting the interpretation of retrospective studies. However, progress has been made to address these research limitations in Nyanza Province, Kenya.

Nyanza Province is located in Western Kenya and, as a high-risk eBL region, has developed a pediatric cancer diagnostic and treatment center at Nyanza Provincial General Hospital (NPGH), thereby facilitating eBL case recognition and reporting. The Province has an average annual eBL incidence rate of 2.15 cases per 100,000 children less than 16 years of age. ${ }^{8}$ Similar to other studies, ${ }^{11}$ we found that eBL

\footnotetext{
* Address correspondence to Ann M. Moormann, Center for Global Health and Diseases, Case Western Reserve University School of Medicine, 10900 Euclid Avenue, Wolstein Research Building 4-130, Cleveland OH, 44106-7286. E-mail: moorms@case.edu
}

cases occur in rural areas of the Province, ${ }^{8}$ primarily in lowland areas around Lake Victoria where malaria transmission is chronic and intense. Within these malarious areas, however, we also detected statistically significant spatial case clustering, reinforcing the potential role of other co-factors. Certain environmental and socio-cultural factors are also likely to cluster within communities and small geographical areas. We conducted a population-based case-control study to explore whether household characteristics, including sibship relationships, could be linked to eBL and its non-random distribution in Nyanza Province. Findings from this exploratory study would be used to direct future eBL research and pediatric cancer awareness campaigns.

\section{MATERIALS AND METHODS}

Study population. This study explored potential eBL cofactors in Nyanza Province, a high-risk eBL region located in Western Kenya (Figure 1). In 1999, roughly 4.5 million persons resided in the Nyanza Province with approximately 50\% of this population less than 15 years of age ${ }^{16}$; the majority of the population resided in rural communities and was involved in subsistence farming. Luo were the predominant tribe (58\%), followed by Kisii (33\%) with (2\%), Kuria (1\%), and Suba (13\%). At the time of this study, Nyanza Province was divided administratively into 12 Districts, 60 Divisions, 324 Locations, and 933 Sub-Locations.

The Province experiences a semi-tropical to tropical climate, with elevations ranging between approximately 1,080 and 2,200 meters. Malaria follows this elevation gradient with holoendemic transmission in the lowland areas around Lake Victoria to sporadic and epidemic transmission in the highlands. ${ }^{17}$ Malaria remains the leading cause of childhood mortality in sub-Saharan Africa. Nyanza Province has also been greatly impacted by HIV/AIDS. Surveys suggest that in Kisumu, the largest urban center in the Province, roughly $27 \%$ of the adult population was HIV seropositive. ${ }^{18}$

Case recruitment. All patients with histologically confirmed eBL at Nyanza Provincial General Hospital (NPGH) 


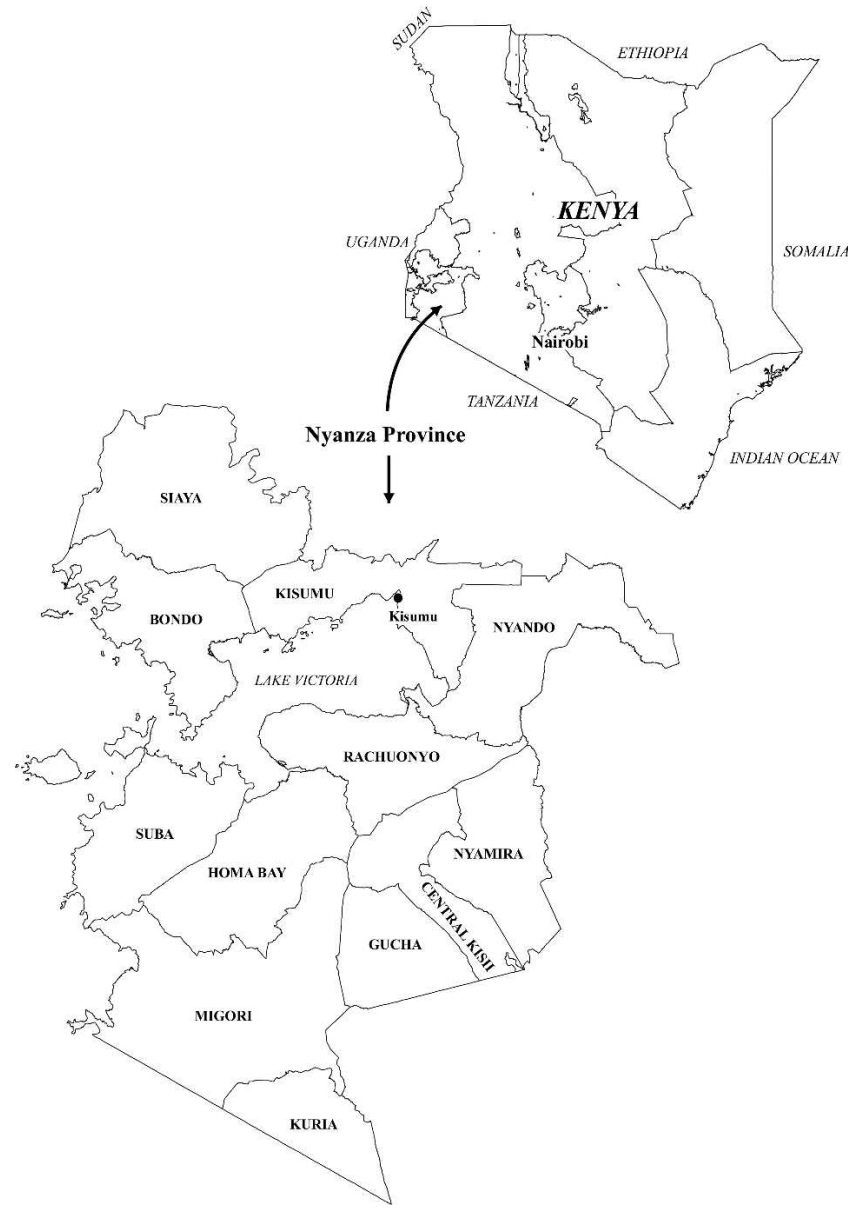

Figure 1. Map of Kenya and district level map of Nyanza Province. The highland area of Nyanza Province includes Nyamira, Central Kisii, and Gucha Districts.

from February through July 2004, who were under 16 years of age, HIV negative, and resided in Nyanza Province for at least 2 years prior to the date of hospitalization were eligible for this study. Cases of eBL who were 16 years of age and older likely reflected a different etiology (HIV-associated), and therefore were not included in this study. The residential requirement limited study participants to those with environmental exposures in Nyanza Province, and was based on the maximum proposed latent period between the final triggering event and tumor development ${ }^{6}$ as well as the approximate time period for eBL occurrence in persons after immigration from low-risk to high-risk eBL regions. ${ }^{19}$

Control recruitment. We selected population-based controls through a multistage cluster-sampling scheme. Thirty Locations and one Sub-Location from each identified Location were selected based on Probability Proportionate to Size (PPS) in the first and second stages, respectively. Selected Sub-Locations were further divided into 1 to 20 Enumeration Areas (EA). A village, city, or town could contain more than one EA (100-150 households), but an EA could not cover more than one village. Therefore, a single EA was selected from each Sub-Location using PPS in our third sampling stage, resulting in 30 identified EAs. Within each EA, three controls were identified and recruited following a random selection process ${ }^{20}$ (one per household and frequency matched to eBL cases according to gender [male, female] and age [2-5, 6-10, 11-15 years of age] categories). Location, SubLocation, and EA population estimates for children 2-15 years of age in 2004 were generated from 1999 census data provided by the Kenya Medical Research Institute/Wellcome Trust Collaborative Program, along with 1999 single-year age estimates and district-level population projections for 2004 obtained from the Kenyan Central Bureau of Statistics.

Data collection. A standardized questionnaire was developed and administered to both cases and controls. We completed interviews and collected observation data during a single home visit for controls. For cases, we conducted a hospital-based interview shortly after each child was admitted to the hospital. Home observation data was obtained after hospital discharge.

Analysis. Data were entered into SAS statistical software program version 9.1 (Cary, NC) for analysis. Observations were weighted according to selection probabilities, response rates, and post-stratification weights. Each case was assigned a selection probability of one. Individual control selection probabilities reflected combined probabilities of the selected Location, Sub-Location, EA, household, and eligible child within a given household. Response rates were $97 \%$ and $98 \%$ for cases and controls, respectively. The final study sample included a greater proportion of controls residing in the highland region compared with cases (Figure 1). Because exposure variables were known to differ in the highlands, we applied post-stratification weights to control responses so that these data conformed proportionately to the geographic distribution (lowland/highland) of cases (i.e., the proportion of cases in each region served as the desired distribution of controls). By dividing each individual weight by the average of control weights combined, control weights were subsequently normalized to maintain the original case-control subject ratio. Standard errors for all statistical estimates were adjusted to compensate for intraclass (within EA) correlation using appropriate procedures in SAS.

We generated crude and confounder adjusted odds ratios (OR) using unconditional logistic regression. Although age and gender were not statistically associated with eBL in our study population, these variables were known eBL risk factors, and were, therefore, included in multivariate models in an effort to control for residual confounding. Mother's tribe was dichotomized into Luo and other, and also included in multivariate models. All live births for the same mother and father were included in birth order and sibship size calculations. We used number of younger siblings (birth order subtracted from total sibship size) to control for sibship size in our analysis of birth order. Effect modification by age and gender was examined through inclusion of appropriate interaction terms to multivariate regression models. All subgroup analyses were conducted using STATA version 9 (College Station, TX) to maintain study design properties (i.e., proper standard error calculations). Results (including case and control counts in tables) reflect statistical adjustments for selection probabilities, response rates, and cluster sampling. As an exploratory study, we used an alpha level of 0.10 to assess Type I errors and generated $90 \%$ confidence intervals for odds ratios. This alpha level, given a small sample size and cluster sampling, allowed us to identify variables that may be of etiological, rather than only statistical, significance. 
This study was approved by the Institutional Review Board at the University of Michigan and at Case Western Reserve University, University Hospitals of Cleveland, and by the Ethical Review Committee at the Kenya Medical Research Institute. We obtained written consent from parents or guardians of all study participants.

\section{RESULTS}

We recruited a total of 41 eBL cases and 91 populationbased controls during the 6-month study period. Seven of the 41 cases $(17 \%)$ were excluded due to residence outside of Nyanza Province. Six of these were from Western Province and one was from Trans Mara District in Rift Valley Province. One case died prior to the interview. Thirty-three eligible cases, therefore, were interviewed and home observations were collected for $20(60 \%)$ of these. Ten $(30 \%)$ cases were interviewed but died during the first 6 weeks of chemotherapy. We did not collect home observations for these children. Inaccessible roads prohibited home observations for three $(9 \%)$ additional cases.

Demographic information of cases and controls participating in the study are presented in Table 1. Age and gender did not differ between cases and controls due to frequency matching, but tribe was statistically associated with participant status. Cases were 3.5 times more likely than controls to be Luo $(\mathrm{OR}=3.51,90 \% \mathrm{CI}: 1.14,10.83)$ even after adjusting (with post-stratification weights) for over-sampling controls from highland regions where the Kisii tend to reside.

Family characteristics. Sibship size for our study population ranged from 1 to 12 children, with a mean of 4.8. The majority $(51 \%)$ of children in the study population had at least one deceased sibling, and a smaller, yet still important percentage, had at least one deceased parent (14\%). Roughly $65 \%$ of participating families reported monogamous parental relationships, but this varied by tribe; the percentage was highest among the Luhya and Kisii while it was lowest in the Luo and Suba, which was consistent with tribal practices. Other family settings included polygamous $(27 \%)$, single parents $(3 \%)$, and living with relatives (6\%). Approximately $43 \%$ of the mothers and fathers had at least some high school education.

We generated crude odds ratios as well as age, gender, and tribe-adjusted odds ratios for birth order, sibship size, family setting, sibling death, parental death, and mother's education

TABLE 1

Demographics of study participants (case and control counts reflect weighting)

\begin{tabular}{lcc}
\hline & Cases & Controls \\
\hline Characteristics—no. $(\%)$ & $(N=34)$ & $(N=91)$ \\
Gender & & \\
$\quad$ Male & $22.67(66.67)$ & $51.22(56.28)$ \\
$\quad$ Female & $11.33(33.33)$ & $39.78(43.72)$ \\
Age group (yrs) & & \\
2-5 & $15.45(45.45)$ & $38.23(42.01)$ \\
6-10 & $12.36(36.36)$ & $30.64(33.67)$ \\
11-15 & $6.18(18.18)$ & $22.13(24.32)$ \\
Tribe (mother) & & \\
Luo & $28.85(84.84)$ & $55.92(61.46)$ \\
Luhya & $1.03(3.03)$ & $7.26(7.98)$ \\
Kisii & $1.03(3.03)$ & $2.66(2.92)$ \\
Other* & $3.09(9.09)$ & $25.16(27.65)$ \\
\hline *Other tribes include Kuria, Suba, Samia, Arab, Mteso, and Mganda.
\end{tabular}

(Table 2). Endemic BL was not statistically associated with either birth order or total sibship size in the crude or adjusted analysis. Because birth order is linked to total sibship size, we used the number of younger siblings (total sibship size minus birth order) to simultaneously analyze both of these factors on eBL risk. Although eBL was not associated with birth order in these adjusted analyses, the impact of having younger siblings on eBL risk was noteworthy. In the multivariate analysis, odds ratios associated with having one and three or more younger siblings compared with none were 0.28 (90\% CI: $0.09,0.83)$ and 0.15 (90\% CI: 0.03, 0.67) regardless of age, gender tribe, and birth order. The other younger sibling comparison (two younger siblings compared with none) was not significant at an alpha of $0.10(\mathrm{OR}=0.59,90 \% \mathrm{CI}$ : $0.16,2.23)$; however, the odds ratio for each group compared with no younger siblings demonstrated a protective effect, suggesting that children with eBL were more likely than controls to be the last born in the family. The age distribution was similar for cases and controls in the total study population $(\mathrm{F}=0.01, P=0.94)$ as well as in each younger sibship category. The observed association with being last-born, therefore, was unlikely due to the residual confounding of age.

Compared with controls, children with eBL were also roughly two times more likely to be residing in a nonmonogamous family setting in the crude analysis. This finding remained statistically significant after adjusting for tribe, age, and gender $(\mathrm{OR}=3.15,90 \% \mathrm{CI}$ : $1.19,8.17)$. Children with eBL were over three times more likely than controls to have at least one deceased parent in the crude analysis $(\mathrm{OR}=3.20$, $90 \%$ CI: 1.08, 9.47). Although this association was not strongly confounded by age, gender, and tribe, the role of parental death was modified by gender (Table 3 ). In the gender-stratified analyses, the tribe and age adjusted OR was 0.99 for males $(90 \%$ CI: $0.23,4.21)$ and 27.99 for females (90\% CI: 2.21, 354.91). The loss of a parent appeared to increase the eBL risk for female children, but not male children. Of the 33 cases, four had a deceased father, one had a deceased mother, and three had both parents deceased. Of the 91 controls, four had a deceased father, three had a deceased mother, and one had lost both parents. Endemic BL was not statistically associated with sibling death or mother's education level.

Physical home environment. We additionally analyzed home observation data. Housing structure was similar for all study participants with the majority of both groups living in homes constructed of mud-wood walls and grass rooftops $(\mathrm{OR}=0.76,90 \% \mathrm{CI}: 0.25,2.01)$ after adjustment for mother's tribe.

\section{DISCUSSION}

Our findings from this exploratory study provide evidence that certain household characteristics, especially sibship relationships, are likely associated with eBL incidence in Nyanza Province. First, eBL cases were more likely than controls to be last-born among their sibship regardless of family size. The third child of three, for example, had an increased risk of eBL as did the seventh child of seven. Second, eBL cases were also more likely than controls to be living in a non-monomagous family setting. Only three $(9 \%)$ of the 33 children with eBL were firstborn and two of these firstborn children were living with relatives or in a polygamous family setting at the time of 
TABLE 2

Risk of endemic Burkitt's lymphoma according to family characteristics (case and control counts reflect weighting)

\begin{tabular}{|c|c|c|c|c|c|c|}
\hline \multirow[b]{2}{*}{ Characteristics } & \multirow[b]{2}{*}{ No. of Cases (\%) } & \multirow[b]{2}{*}{ No. of Controls (\%) } & \multicolumn{2}{|c|}{ Crude } & \multicolumn{2}{|c|}{ Adjusted ${ }^{*}$} \\
\hline & & & OR & $90 \% \mathrm{CI}$ & OR & $90 \% \mathrm{CI}$ \\
\hline \multicolumn{7}{|l|}{ Sibship size } \\
\hline $1-2$ & $5.15(15.15)$ & $18.37(20.19)$ & 1.00 & - & 1.00 & - \\
\hline $3-4$ & $11.33(33.33)$ & $26.71(29.37)$ & 1.51 & $0.46,4.98$ & 1.47 & $0.47,4.58$ \\
\hline $5-6$ & $10.30(30.30)$ & $22.13(24.32)$ & 1.67 & $0.53,5.20$ & 1.91 & $0.61,5.97$ \\
\hline $7+$ & $7.21(21.21)$ & $23.79(26.14)$ & 1.08 & $0.30,3.85$ & 1.01 & $0.29,3.48$ \\
\hline \multicolumn{7}{|l|}{ Birth order } \\
\hline 1 & $6.18(18.18)$ & $32.04(35.21)$ & 1.00 & - & 1.00 & - \\
\hline 2 & $5.15(15.15)$ & 10.09 (11.09) & 2.65 & $0.65,10.82$ & 2.92 & $0.59,14.42$ \\
\hline 3 & $8.24(24.24)$ & $6.04(6.64)$ & 7.08 & $2.27,22.03$ & 6.79 & $1.77,26.16$ \\
\hline 4 & $3.09(9.09)$ & $16.33(17.95)$ & 0.98 & $0.25,3.89$ & 0.96 & $0.22,4.17$ \\
\hline $5+$ & $11.33(33.33)$ & $32.04(35.21)$ & 2.22 & $0.72,6.83$ & 1.86 & $0.59,5.91$ \\
\hline \multicolumn{7}{|l|}{ No. of younger siblings } \\
\hline 0 & $12.36(36.36)$ & $21.74(23.89)$ & 1.00 & - & 1.00 & - \\
\hline 1 & $8.24(24.24)$ & $32.09(35.27)$ & 0.45 & $0.16,1.26$ & 0.28 & $0.09,0.83$ \\
\hline 2 & $10.30(30.30)$ & $17.15(18.84)$ & 1.06 & $0.37,3.05$ & 0.59 & $0.16,2.23$ \\
\hline $3+$ & $3.09(9.09)$ & $20.22(21.99)$ & 0.27 & $0.08,0.96$ & 0.15 & $0.03,0.67$ \\
\hline \multicolumn{7}{|l|}{ Death of $>=1$ sibling } \\
\hline No & $21.63(63.63)$ & $52.39(57.57)$ & 1.00 & - & 1.00 & - \\
\hline Yes & $12.36(36.36)$ & $38.61(42.42)$ & 0.78 & $0.36,1.68$ & 0.70 & $0.30,1.66$ \\
\hline \multicolumn{7}{|l|}{ Family setting** } \\
\hline Monogamous & $16.48(48.48)$ & $60.91(66.93)$ & 1.00 & - & 1.00 & - \\
\hline Other & $17.52(51.52)$ & $30.09(33.07)$ & 2.15 & $0.92,5.04$ & 3.12 & $1.19,8.17$ \\
\hline \multicolumn{7}{|l|}{ Death of $>=1$ parent* } \\
\hline No & $24.73(75.00)$ & $82.42(90.57)$ & 1.00 & - & 1.00 & - \\
\hline Yes & $8.24(25.00)$ & $8.58(9.43)$ & 3.20 & $1.08,9.47$ & 3.38 & $1.18,9.64$ \\
\hline \multicolumn{7}{|l|}{ Mother's education** } \\
\hline Some primary or less & $15.45(48.39)$ & $35.84(40.99)$ & 1.00 & - & 1.00 & - \\
\hline Some high school & $13.40(41.94)$ & $39.32(44.96)$ & 0.58 & $0.18,1.93$ & 0.78 & $0.35,1.76$ \\
\hline High school or more & $3.09(9.68)$ & $12.29(14.05)$ & 0.79 & $0.38,1.65$ & 0.80 & $0.24,2.59$ \\
\hline
\end{tabular}

Adjusted for age, gender, and mother's tribe.

-Birth order also adjusted for number of younger siblings, and number of younger siblings adjusted for birth order.

*** Frequencies and odds ratios exclude unknown responses, other includes polygamous family setting, single parent and living with relatives.

onset. Third, children with eBL were three times more likely to have a deceased parent compared with healthy children. Roughly $24 \%$ of cases and $8 \%$ of controls had at least one deceased parent.

The mechanisms through which these variables might operate to increase the risk of eBL are currently unknown and will require further investigation. However, birth order often serves as a proxy for exposure to infectious diseases early in life. Guy de Thé postulated that early infection with EBV was a risk factor for eBL. ${ }^{1}$ Our data support this hypothesis, as last-born children would have greater chance of exposure to infectious agents shed by their older siblings and are thus more likely to be infected with EBV earlier in life than older children. Alternatively, co-infection with other pathogens at the time of primary EBV infection could also be a factor. Malaria-induced immunosuppression of EBV-specific T-cell immunosurveillance has been suggested as a mechanism that increases a child's risk of developing eBL. ${ }^{21-23}$ Last-born children may also experience greater competition for limited resources that result in inadequate nutrition. Clifford attributed increased risk of eBL among low families with low socioeconomic status (SES) to protein deficiency. ${ }^{24}$ Poor nutritional status is known to decrease immunosurveillance, which could increase the opportunity for an undetected oncogenic event to occur. ${ }^{2}$

The observed increased risk of living in a non-monogamous setting (polygamous family or living with relatives) as well as having a deceased parent may be similarly related to limited resources or increased exposure to infectious agents. ${ }^{2}$ The association between eBL and having a deceased parent differed by gender; death of parent increasing the risk of eBL among female but not male children. Loss of a parent typi-

TABLE 3

Parent's death on risk of endemic Burkitt's lymphoma by gender (case and control counts reflect weighting)

\begin{tabular}{|c|c|c|c|c|c|c|}
\hline \multirow[b]{2}{*}{ Death of $>=1$ parent $~$} & \multirow[b]{2}{*}{ No. of Cases } & \multirow[b]{2}{*}{ No. of Controls } & \multicolumn{2}{|c|}{ Crude } & \multicolumn{2}{|c|}{ Adjusted* } \\
\hline & & & OR & $90 \% \mathrm{CI}$ & OR & $90 \% \mathrm{CI}$ \\
\hline \multicolumn{7}{|l|}{ Male } \\
\hline No & $18.55(85.71)$ & $43.84(85.59)$ & 1.00 & - & 1.00 & - \\
\hline Yes & 3.09 (14.29) & $7.38(14.40)$ & 0.99 & $0.23,4.21$ & 0.99 & $0.25,3.92$ \\
\hline \multicolumn{7}{|l|}{ Female } \\
\hline No & $6.18(54.55)$ & $38.57(96.96)$ & 1.00 & - & 1.00 & - \\
\hline Yes & $5.15(45.45)$ & $1.21(3.03)$ & 26.62 & $3.67,193.05$ & 42.21 & $2.06,888.43$ \\
\hline
\end{tabular}

Frequencies and odds ratios exclude unknown responses. 
cally results in a decrease of resources, and this may disproportionately affect female rather than male children. ${ }^{24}$ Due to the small number of cases $(N=11)$, however, these findings should be interpreted cautiously. Future investigations of child's age as well as length of time since parent's death may provide a better understanding of this association. Although we lacked information to substantiate the cause of parents' death, all eBL cases were HIV negative, suggesting that HIV did not directly influence eBL incidence. However, the prevalence of HIV/AIDS is relatively high in our study area and has resulted in an increased rate of parental death and lower life expectancy. ${ }^{18}$

This is the first study to examine the role of birth order on eBL, and although being last-born was significant in our study, overall sibship size was not. This differs with an earlier study conducted by Morrow who detected an increase in eBL risk among children with a larger sibship size. ${ }^{25}$ Both studies suggest a role of sibship on eBL risk, and the discrepancy between our findings and Morrow's may reflect different household and sibship definitions as well as sampling variability, including differences in sibship spacing and gender distribution. Nevertheless, birth order as a risk factor has been reported for other childhood cancers, including childhood acute lymphoblastic leukemia and acute myeloid leukemia. ${ }^{15,26}$ In addition, a large multicenter US case-control study of Non-Hodgkin's Lymphoma (NHL), found an increased risk for NHL, in particular diffuse large B-cell lymphoma, among last-born children, with a much smaller effect of large sibship size (Cozen, personal communication). Our study is limited by the small sample size. However, given that our results are consistent with other studies showing an increased risk for cancer associated with being last-born, a common etiologic mechanism is suggested.

Based on the physical structure of the home, cases and controls did not differ significantly by socio-economic status, a variable associated with eBL in other studies. ${ }^{9,27}$ We defined housing structure variables according to the building unit in which the study participant most often slept. Data on other units in the compound and other economic variables, including ownership of radios, bicycles, and vehicles, were not collected. Although we recruited eBL cases following hospital admission, there was no evidence to suggest that cases had better health care access compared with population-based controls. Many of the eBL cases reaching Nyanza Provincial hospital resided in isolated regions of the Province. The study population included three cases from Suba District, a region with limited infrastructure in the south-western region of Nyanza Province, and four cases from Migori District, with some of the most remote communities in Nyanza. Regardless of economic standing and transportation constraints, the majority of eBL cases presented directly at NGPH or was referred to the treatment center by a local health authority. Cases and controls appeared to be socio-economically comparable. Additional data and a more rigorous analysis would be needed to thoroughly examine the socio-economic variability between study groups. Data collected in this study reflected only crude estimates of SES status.

To examine multiple eBL co-factors, we conducted a population-based case-control study. Because NPGH is the regional referral and treatment center for all childhood cancers within the Province, this clearly facilitated case identification. However, because NPGH is also a local treatment hospital, using other hospitalized patients as a control population was not feasible. Because of these challenges, we opted to select controls from the healthy childhood population living in the Province within the hospital referral area. Despite the lack of a sampling frame, we were able to select controls randomly from the disease-free at-risk population in the study area using a multistage cluster-sampling (area probability) scheme. Limitations to our study design included the use of cluster sampling, which frequently leads to increases in standard error estimates and wider confidence intervals. Additionally, post-stratification weights were applied to all control values. These weights adjusted for a disproportional number of controls selected from the highland region of the Province compared with cases, thereby minimizing potential confounding due to tribe and related genetic and environmental factors associated with the Kisii who predominantly live in the highlands. Although our results were valid, the efficiency of our study was decreased. Application of stratification techniques in the design phase of the study could have greatly improved study efficiency and maximized data collection efforts. Future studies will include these design changes.

Exposure to chronic and intense malaria transmission has long been considered an eBL risk factor. In our previous investigations, however, we identified several high-risk clusters of eBL within the holodendemic regions of the Province. ${ }^{8}$ Morrow, based on his early work in Uganda, suggested that the distribution of eBL within holoendemic malaria areas was likely linked to small area variability in malaria transmission intensity. ${ }^{3}$ Although malaria transmission intensity varies significantly between the highland and lowland regions of the Province, current data is lacking regarding small area variability within the lowland areas. Despite potential transmission variability within these high-risk clusters, we proposed that other eBL co-factors, which may operate alone or along with malaria to further decrease antiviral immunity, are likely involved. This study allowed us to explore some of these additional co-factors, including other environmental and sociocultural factors, which may also cluster at the local level.

Endemic BL has been cited as a classic model of multi-step tumorigenesis. The present paradigm for the etiology of eBL starts with early-age EBV infection, followed by subsequent repeated malaria infections that suppress EBV-specific immunity and culminates in tumorigenesis (reviewed in ${ }^{13}$ ). We found that being last-born increased the risk for eBL. Although the mechanisms through which familial factors operate to increase eBL risk are currently unknown, early age of EBV infection, increased competition for resources and ultimately poor nutritional status, or impact of birth order on host immune responses may be contributing to increased risk. This initial work on family environmental factors will contribute to future eBL research and provides novel insight into multi-factorial eBL etiology.

Received April 10, 2007. Accepted for publication October 23, 2007.

Acknowledgments: The success of this project relied on the dedicated work of Juliana Otieno, Chief Pediatrician at Nyanza Provincial General Hospital, who diagnosed and treated the Burkitt's cases included in this study. We also thank Dorine Omenah and Steve Obara at the Kenya Medical Research Institute in Kisumu, Kenya for their work in study participant recruitment and Brady West of the Center for Statistical Consultation and Research at the University of Michigan for his statistical advice. This manuscript was approved by the Director of the Kenya Medical Research Institute. 
This project was supported by the Munn Idea Grant of the Comprehensive Cancer Center, Rackham Graduate School and the Center for Global Health at the University of Michigan, and from NIH Grant K08 AI051565 "Immunologic studies of endemic Burkitt's lymphoma" (Moormann).

Authors' addresses: Jeanette J. Rainey, Global Immunization Division, National Center for Immunization and Respiratory Diseases, Centers for Disease Control and Prevention, Mailstop E-05 1600 Clifton Road NE, Atlanta, GA 30333, Tel: (404) 639-8474, Fax: (404) 639-8676, E-mail: jkr7@cdc.gov. Rosemary Rochford, Department of Microbiology and Immunology, SUNY Upstate Medical University, 750 East Adams St., Syracuse, NY 13210, Tel: (315) 464-5468, Fax: (315) 464-4417, E-mail: rochforr@upstate.edu. Peter Odada Sumba, Center for Vector Biology \& Control Research, Kenya Medical Research Institute PO Box 1578, Kisumu, Kenya, Tel: 254-57-202-2989, E-mail: POdada@kisian.mimcom.net. Dickens Kowuor, Center for Vector Biology \& Control Research, Kenya Medical Research Institute, PO Box 1578, Kisumu, Kenya, Tel: 254-57-202-2989, E-mail: DKowuor@kisian.mimcom.net. Mark L. Wilson, Global Health, Department of Epidemiology, School of Public Health, University of Michigan, 109 Observatory St., Room 2006, Ann Arbor, MI 481092029, Tel: (734) 936-0152, Fax: (734) 764-3192, E-mail: wilsonml@ umich.edu. Ann M. Moormann, Center for Global Health \& Diseases, Case Western Reserve University, 2103 Cornell Rd 4-130, Wolstein Research Building, Cleveland, OH 44106-7286, Tel: (216) 368-5144, Fax: (216) 368-4882, E-mail: moorms@case.edu.

\section{REFERENCES}

1. de-The G, Geser A, Day NE, Tukei PM, Williams EH, Beri DP, Smith PG, Dean AG, Bronkamm GW, Feorino P, Henle W, 1978. Epidemiological evidence for causal relationship between Epstein-Barr virus and Burkitt's lymphoma from Ugandan prospective study. Nature 274: 756-761.

2. Evans AS, 1985. Epidemiology of Burkitt's lymphoma: other risk factors. IARC Sci Publ 60: 197-204.

3. Morrow RH Jr, 1985. Epidemiological evidence for the role of falciparum malaria in the pathogenesis of Burkitt's lymphoma. IARC Sci Publ 60: 177-186.

4. Henle W, Henle G, 1980. Epidemiologic aspects of Epstein-Barr virus (EBV)-associated diseases. Ann N Y Acad Sci 354: 326331.

5. Moormann AM, Chelimo K, Sumba OP, Lutzke ML, PloutzSnyder R, Newton D, Kazura J, Rochford R, 2005. Exposure to holoendemic malaria results in elevated Epstein-Barr virus loads in children. J Infect Dis 191: 1233-1238.

6. Day NE, Smith PG, Lachet B, 1985. The latent period of Burkitt's lymphoma: the evidence from epidemiological clustering. IARC Sci Publ 60: 187-195.

7. Mwanda OW, Rochford R, Moormann AM, Macneil A, Whalen C, Wilson ML, 2004. Burkitt's lymphoma in Kenya: geographical, age, gender and ethnic distribution. East Afr Med J 8 Suppl: S68-S77.

8. Rainey JJ, Omenah D, Sumba PO, Moormann AM, Rochford R, Wilson ML, 2007. Spatial clustering of endemic Burkitt's lymphoma in high-risk regions of Kenya. Int J Cancer 120: 121127.

9. Williams CK, 1988. Clustering of Burkitt's lymphoma and other high-grade malignant lymphoproliferative diseases, but not acute lymphoblastic leukaemia among socio-economically deprived Nigerians. East Afr Med J 65: 253-263.

10. Griffin BE, 2000. Epstein-Barr virus (EBV) and human disease: facts, opinions and problems. Mutat Res 462: 395-405.

11. Morrow RH, Pike MC, Smith PG, Ziegler JL, Kisuule A, 1971. Burkitt's lymphoma: a time-space cluster of cases in Bwamba County of Uganda. BMJ 2: 491-492.

12. Pike MC, Williams EH, Wright B, 1967. Burkitt's tumour in the West Nile District Uganda, 1961-5. BMJ 2: 395-399.

13. Rochford R, Cannon MJ, Moormann AM, 2005. Endemic Burkitt's lymphoma: a polymicrobial disease? Nat Rev Microbiol 3: 182-187.

14. van den Bosch C, Griffiin BE, Kazembe P, Dziweni C, Kadzamira L, 1993. Are plant factors a missing link in the evolution of endemic Burkitt's lymphoma? Br J Cancer 68: 1232-1235.

15. Westergaard T, Andersen PK, Pedersen JB, Olsen JH, Frisch M, Sorensen HT, Wohlfahrt J, Melbye M, 1997. Birth characteristics, sibling patterns, and acute leukemia risk in childhood: a population-based cohort study. J Natl Cancer Inst 89: 939-947.

16. Kenya Ministry of Planning and National Development, Census Bureau of Statistics, The 1999 Census. Republic of Kenya (2002).

17. Omumbo J, Ouma J, Rapuoda B, Craig MH, le Sueur D, Snow RW, 1998. Mapping malaria transmission intensity using geographical information systems (GIS): an example from Kenya. Ann Trop Med Parasitol 92: 7-21.

18. United States Census Bureau, International Programs Center. Population Division, HIV/AID Profile: Kenya 2000.

19. Mwanda OW, 2004. Clinical characteristics of Burkitt's lymphoma seen in Kenyan patients. East Afr Med J 8 Suppl: S78S89.

20. Crampin AC, Mwinuka V, Malema SS, Glynn JR, Fine PE, 2001. Field-based random sampling without a sampling frame: control selection for a case-control study in rural Africa. Trans $R$ Soc Trop Med Hyg 95: 481-483.

21. Moormann AM, Chelimo K, Sumba PO, Tisch DJ, Rochford R, Kazura JW, 2007. Exposure to holoendemic malaria results in suppression of Epstein-Barr virus-specific $\mathrm{T}$ cell immunosurveillance in Kenyan children. J Infect Dis 195: 799-808.

22. Moss DJ, Burrows SR, Castelino DJ, Kane RG, Pope JH, Rickinson AB, Alpers MP, Heywood PF, 1983. A comparison of Epstein-Barr virus-specific T-cell immunity in malariaendemic and -nonendemic regions of Papua New Guinea. Int $J$ Cancer 31: 727-732.

23. Whittle HC, Brown J, Marsh K, Greenwood BM, Seidelin P, Tighe H, Wedderburn L, 1984. T-cell control of Epstein-Barr virus-infected $\mathrm{B}$ cells is lost during P. falciparum malaria. $\mathrm{Na}$ ture 312: 449-450.

24. Clifford P, Singh S, Stjernsward J, Klein G, 1967. Long-term survival of patients with Burkitt's lymphoma: an assessment of treatment and other factors which may relate to survival. Cancer Res 27: 2578-2615.

25. Morrow RH, Kisuule A, Mafigiri J, 1974. Socioeconomic factors in Burkitt's lymphoma. Cancer Res 34: 1212.

26. Altieri A, Castro F, Bermejo JL, Hemminki K, 2006. Number of siblings and the risk of lymphoma, leukemia, and myeloma by histopathology. Cancer Epidemiol Biomarkers Prev 15: 12811286.

27. Makata AM, Toriyama K, Kamidigo NO, Eto H, Itakura H, 1996. The pattern of pediatric solid malignant tumors in western Kenya, east Africa, 1979-1994: an analysis based on histopathologic study. Am J Trop Med Hyg 54: 343-347. 